Europhysics Letters

PREPRINT

\title{
Oblique electromagnetic instabilities for an ultra relativis- tic electron beam passing through a plasma
}

\author{
A. BRET \\ ETSI Industriales, Universidad de Castilla-La Mancha, 13071 Ciudad Real, Spain
}

PACS. 52.35.Qz - Micro instabilities.

PACS. 52.40.Mj - Particle beam interactions in plasmas.

PACS. 52.35.Hr -Electromagnetic waves.

\begin{abstract}
We present an investigation of the electromagnetic instabilities which are triggered when an ultra relativistic electron beam passes through a plasma. The linear growth rate is computed for every direction of propagation of the unstable modes, and temperatures are modelled using simple waterbag distribution functions. The ultra relativistic unstable spectrum is located around a very narrow band centered on a critical angle which value is given analytically. The growth rate of modes propagating in this direction decreases like $k^{-1 / 3}$.
\end{abstract}

The interaction of a relativistic electron beam with a plasma is a subject of relevance from many fields of physics ranging from inertial confinement fusion [1] to some astrophysical scenarios [2-4]. The linear analysis of the interaction reveals an highly unstable situation which has been investigated for a long time. Modes propagating along the beam are unstable within a certain range of wave-vector and form the electrostatic two-stream instability. The so-called filamentation instability is found for modes propagating transversely to the beam and finally, it can be proved that some modes propagating at arbitrary angle to the beam are also unstable [5]. As far as the growth rate is concerned, we see that it is eventually a function of the parallel and perpendicular wave vector components. As long as the beam is not relativistic, the largest growth rate are found for wave vectors parallel to the beam which means that the two-stream instability dominates the linear evolution of the system in this regime [6]. The situation evolves when considering a relativistic electron beam. Because relativistic electrons are harder to move in the direction of their motion than in the transverse direction, the two-stream growth rate is much more reduced than the growth rate of the modes propagating transversely, or even obliquely. If we denote $\gamma_{b}$ the beam relativistic factor, the maximum two-stream growth rate is scaled like $\gamma_{b}^{-1}$, the filamentation growth rate like $\gamma_{b}^{-1 / 2}$ whereas the growth rate of the most unstable oblique wave vector is scaled like $\gamma_{b}^{-1 / 3}[5]$. This shows that oblique instabilities should dominate all the more than the beam is relativistic. The ultra relativistic limit is relevant for astrophysical settings such as high energy cosmic rays or gamma ray bursts production scenarios, for which some authors consider relativistic factors up to $10^{2}[2]$ and even $10^{7}[3]$. These unstable oblique modes were first investigated in the cold limit (fluid approximation), and a temperature dependant treatment has only been developed recently [7]. As we shall see in this letter, accounting for temperatures in the beam

(C) EDP Sciences 


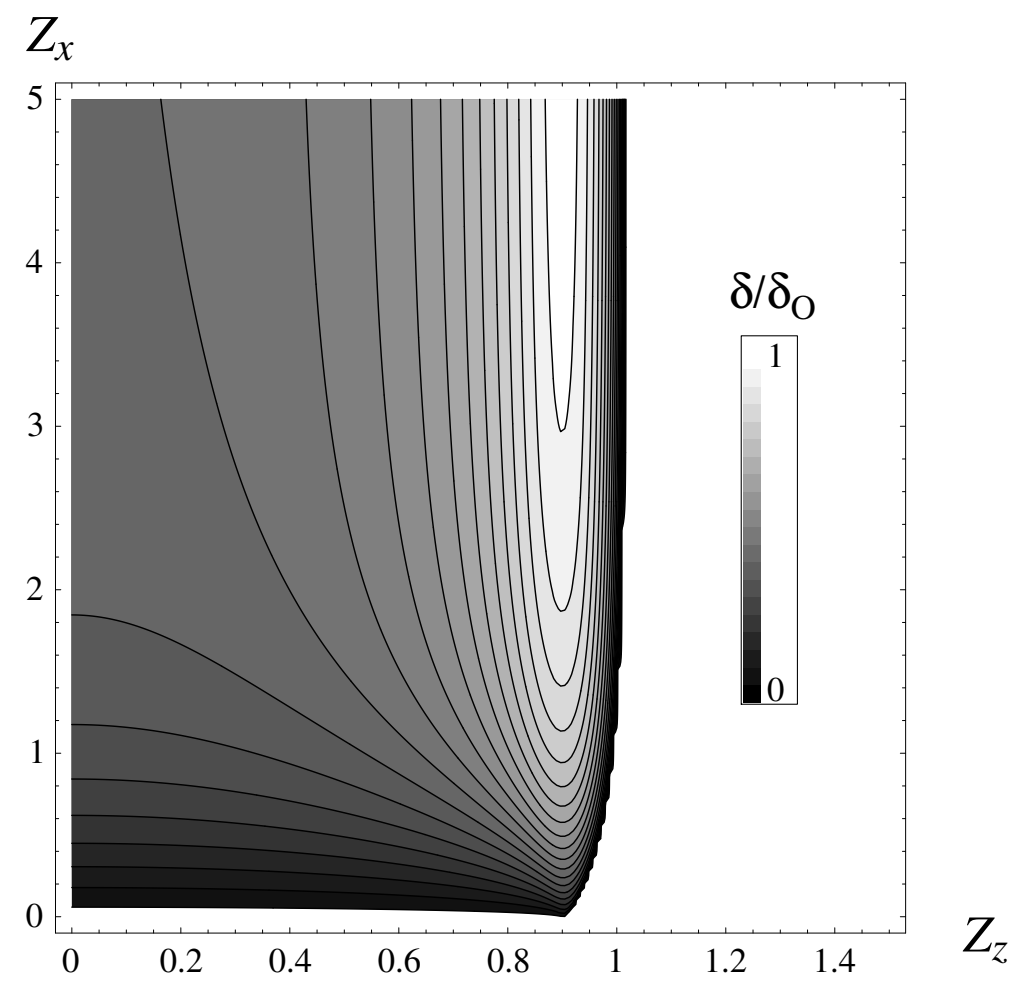

Fig. 1 - Numerical evaluation of the growth rate for a cold beam and a cold plasma, in terms of $\mathbf{Z}=\mathbf{k} V_{b} / \omega_{p p}$. The growth rate is normalized to its maximum value $\delta_{O}$ given by eqs. (3). Parameters are $n_{b} / n_{p}=0.1$ and $\gamma_{b}=200$.

and the plasma results in a very narrow oblique unstable spectrum in the ultra relativistic limit.

We thus consider a relativistic electron beam of velocity $\mathbf{V}_{b}=V_{b} \mathbf{e}_{z}$, gamma factor $\gamma_{b}=$ $1 /\left(1-V_{b}^{2} / c^{2}\right)^{1 / 2}$ and density $n_{b}$ passing through a plasma at electronic density $n_{p} \gg n_{b}$. Ions are supposed to form a fixed neutralizing background and we account for a plasma return neutralizing current [8] at velocity $V_{p}$ such as $n_{b} V_{b}=n_{p} V_{p}$. The system is therefore charge and current neutralized, and we study the stability of harmonic perturbations $\propto \exp (i \mathbf{k} \cdot \mathbf{r}-i \omega)$. We implement a 3D formalism using the linearized relativistic Vlasov equation as well as Maxwell's ones. Given the symmetries of the problem, the wave vector can be chosen within the $(x, z)$ plan, and the dispersion equation reads [9]

$$
\left(\omega^{2} \varepsilon_{x x}-k_{z}^{2} c^{2}\right)\left(\omega^{2} \varepsilon_{z z}-k_{x}^{2} c^{2}\right)-\left(\omega^{2} \varepsilon_{x z}+k_{z} k_{x} c^{2}\right)^{2}=0,
$$

in terms of the dielectric tensor elements

$$
\varepsilon_{l m}=\delta_{l m}+\sum_{j=p, b} \frac{\omega_{p j}^{2}}{n_{j} \omega^{2}} \int \frac{p_{l}}{\gamma} \frac{\partial f_{0 j}}{\partial p_{m}} d^{3} p+\frac{\omega_{p j}^{2}}{n_{j} \omega^{2}} \int \frac{p_{l} p_{m}}{\gamma^{2}} \frac{\mathbf{k} \cdot \partial f_{0 j} / \partial \mathbf{p}}{m \omega-\mathbf{k} \cdot \mathbf{p} / \gamma} d^{3} p,
$$

where $f_{0 p}$ and $f_{0 b}$ are the equilibrium distribution functions for the plasma and the beam, $m$ the electron mass, $\omega_{p p, b}$ the electronic plasma frequencies for the plasma and the beam, and 
$\gamma=\left(1+p^{2} / m^{2} c^{2}\right)^{1 / 2}$. The dispersion equation (1) bridges between the two-stream modes at $k_{x}=0$ and the filamentation ones at $k_{z}=0$. Because the former modes are longitudinal $(\mathbf{k} \| \mathbf{E})$ while the later are purely transverse $(\mathbf{k} \cdot \mathbf{E}=0)$, unstable oblique modes are neither longitudinal nor transverse and a fully electromagnetic dispersion equation such as (1) is required to reach them.

If we start considering a cold mono energetic beam entering a cold plasma, the resulting growth rate map is the one pictured on figure 11 We plot the growth rate normalized to the maximum oblique one in terms of the reduced wave vector $\mathbf{Z}=\mathbf{k} V_{b} / \omega_{p p}$. In this cold limit, the maximum two-stream, filamentation, and oblique growth rates read

$$
\delta_{T S} \sim \frac{\sqrt{3}}{2^{4 / 3}} \frac{\left(n_{b} / n_{p}\right)^{1 / 3}}{\gamma_{b}}, \quad \delta_{F} \sim \sqrt{\frac{n_{b} / n_{p}}{\gamma_{b}}}, \quad \delta_{O} \sim \frac{\sqrt{3}}{2^{4 / 3}}\left(\frac{n_{b} / n_{p}}{\gamma_{b}}\right)^{1 / 3} .
$$

One can check that the two-stream instability is negligible compared to the oblique and the normal modes. We also note on fig. 11 that the most unstable modes form a continuum at $Z_{z} \sim 0.9$, starting from $Z_{x}>3$.

The introduction of temperatures modify the picture in various ways. As long as they remain "small", they can be modelled through some simple waterbag distributions and the tensor elements (2) can be calculated exactly [7]. It turns out that the resulting dispersion function has various singularities corresponding to various kind of preferential coupling between a mode and some electron population. As the wave vector is more and more oblique, these singularities evolve and two of them overlap for a critical inclination angle $\theta_{c}$ with the beam, which ultra relativistic expression is simply

$$
\tan \theta_{c}=\frac{1+n_{b} / n_{p}}{V_{t p} / V_{b}}
$$

where $V_{t p}$ is the plasma thermal velocity. At this junction, let us comment what is meant by "small temperatures". As far as the plasma temperature is concerned, it simply means that the thermal velocity must remains small compared to the beam one. The present analysis is therefore valid for non-relativistic plasma temperatures, allowing for a $10 \mathrm{keV}$ plasma for example. As far as beam temperature is concerned, transverse and parallel temperatures can be treated differently. Let us assume a waterbag distribution function defining a momentum spread around $P_{b}=\gamma_{b} m V_{b}$,

$$
f_{0 b}=\frac{n_{b}}{4 P_{t b \perp} P_{t b \|}}\left[\Theta\left(p z-P_{b}+P_{t b \|}\right)-\Theta\left(p z+P_{b}+P_{t b \|}\right)\right]\left[\Theta\left(p_{x}+P_{t b \perp}\right)-\Theta\left(p_{x}-P_{t b \perp}\right)\right]
$$

where $\Theta$ is the step function. As can be seen on eqs. (2), the tensor elements are mostly dependent of the velocity distribution through the quantities $\mathbf{p} / \gamma$, and this is perfectly expected since the stability of a mode is a matter a wave particle interaction. Let us then evaluate the velocity spread $\left(\Delta v_{\|}, \Delta v_{\perp}\right)$ corresponding to the momentum spread $\left(P_{t b \|}, P_{t b \perp}\right)$ defined above. In the present ultra relativistic regime, we find

$$
\Delta v_{\|} \sim \frac{1}{\gamma_{b}^{2}} \frac{\Delta P_{t b \|}}{m \gamma_{b}}, \quad \Delta v_{\perp} \sim \frac{P_{t b \perp}}{m \gamma_{b}}
$$

We recover for $\Delta v_{\perp}$ the velocity spread corresponding to the momentum spread $P_{t b \perp}$. But $\Delta v_{\|}$is reduced by a factor $\gamma_{b}^{2}$ so that the parallel beam temperature can be neglected in the very large $\gamma_{b}$ limit. 


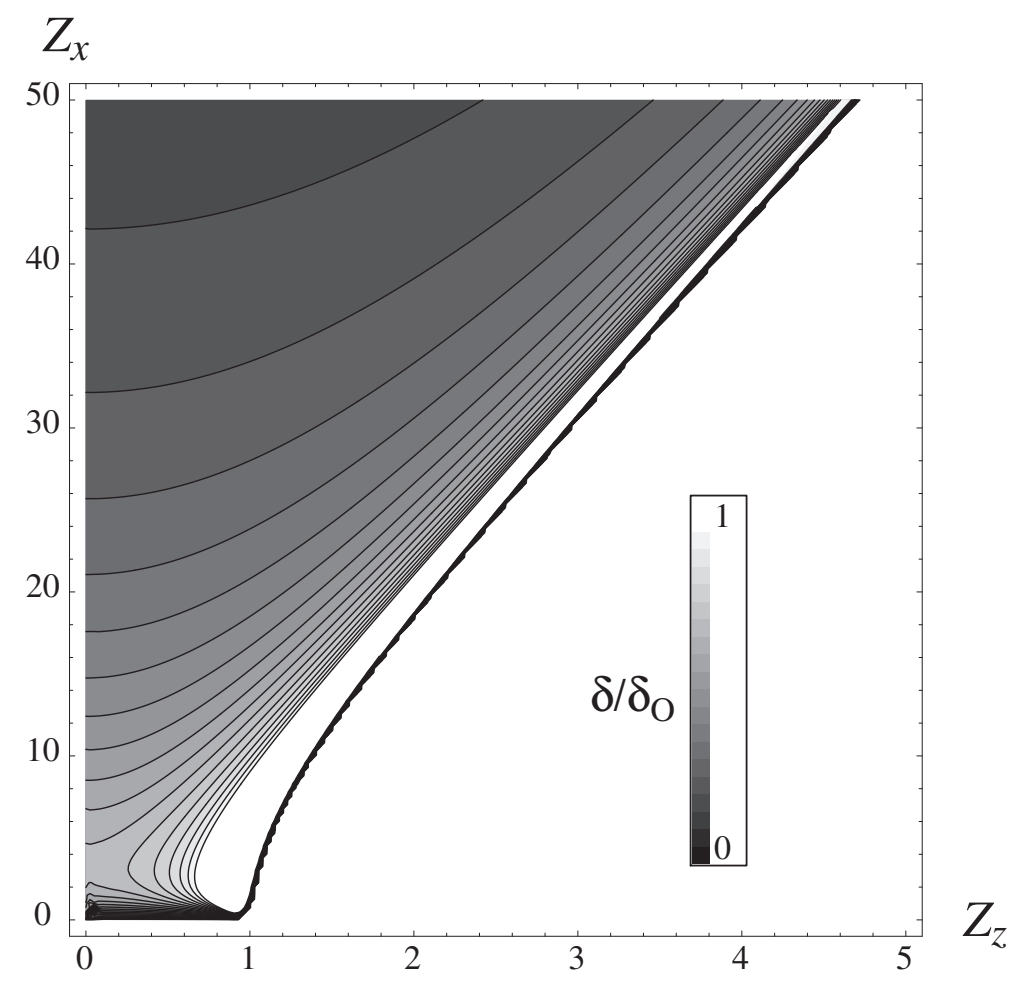

Fig. 2 - Numerical evaluation of the growth rate for a hot beam and a hot plasma, in terms of $\mathbf{Z}=\mathbf{k} V_{b} / \omega_{p p}$. The growth rate is normalized to its maximum value $\delta_{O}$ given by eqs. (3). Parameters are $n_{b} / n_{p}=0.1$ and $\gamma_{b}=200$. The parallel beam velocity has been set to 0 , and every other thermal velocities are $c / 10$.

Considering a relativistic beam with $\gamma_{b}=200, n_{b}=n_{p} / 10$ and neglecting its parallel thermal spread, we plot on fig. 22 the growth rate in terms of $\mathbf{Z}=\mathbf{k} V_{b} / \omega_{p p}$. The continuum of the most unstable modes observed on fig. 1 has turned into a very thin oblique region where the growth rate is higher than $90 \%$ of its cold oblique value $\delta_{O}$ given by eq. (3) up to $Z \sim 50$. Let us emphasize that unlike fig. 1 where the largest reduced wave vector plotted is $Z_{z}=1.5$ and $Z_{x}=5$, we here extend the plot up to $Z_{z}=5$ and $Z_{x}=50$. Modes located above the critical angle are unstable up to $Z_{x} \sim \gamma_{b} /\left(V_{t p} / V_{b}\right)+Z_{z} \tan \theta_{c}$ [7]. We also note that the transition towards stable modes bellow the critical angle is very sharp so that the instability domain is almost rigourously bounded by the directions $\theta_{c}$ and $\pi / 2$. The critical angle obtained from eq. (4) is $\arctan (11) \sim 84.8^{\circ}$, which corresponds very precisely to what is observed on the graph. In order to study the growth rate at large $Z$ in the critical direction, we plot on figure 3 the maximum growth rate at angle $\theta_{c}$ for $Z$ up to $10^{4}$. We observe a decrease with $Z$ which can presently be fitted by $\delta / \delta_{O}=2.1 Z^{-1 / 3}$. It is interesting to notice that the maximum filamentation growth rate is here $\delta_{F}=0.41 \delta_{O}$ so that the growth rate in the critical direction remains larger than the filamentation one until $Z \sim 150$.

In conclusion, we have evaluated the growth rate of the electromagnetic instabilities for an ultra-relativistic beam passing through a non-relativistic plasma for any angle of propagation of the unstable modes. The parallel temperature of the beam can be neglected, and the 


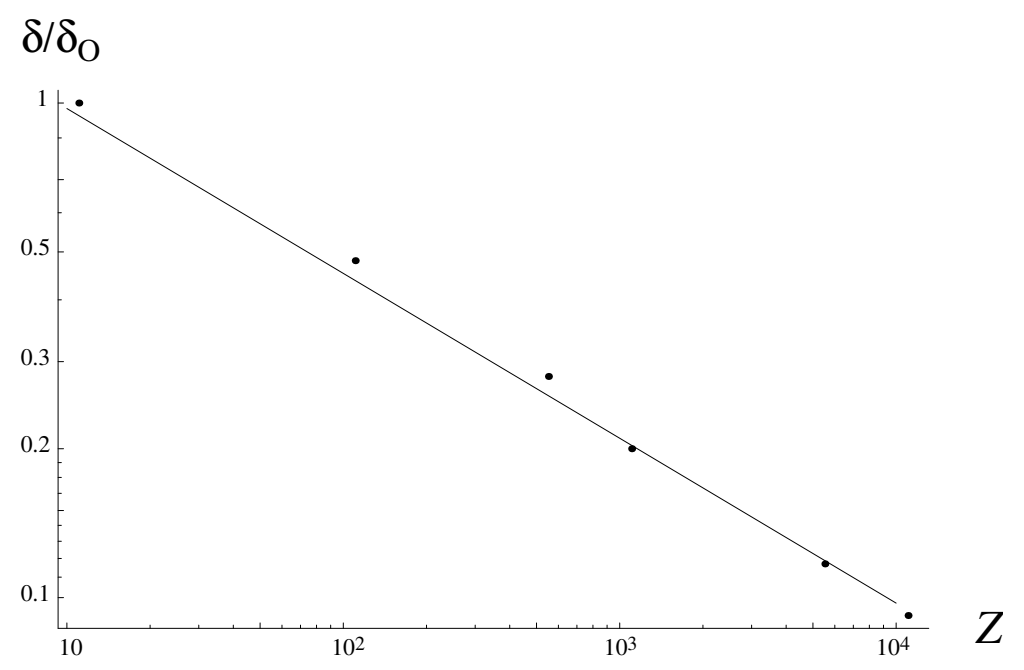

Fig. 3 - Numerical evaluation of the growth rate in the critical direction up to $Z=10^{4}$. Same parameters as fig. 2 The black points are the result of numerical calculation and the line represents $\delta / \delta_{O}=2.1 Z^{-1 / 3}$.

unstable waves are found in a narrow domain comprised between $\pi / 2$ and the critical angle $\theta_{c}$ which does not depends on the beam energy (see eq. 44). Within this region, the most unstable modes are concentrated on a very narrow band extending around the critical direction, with a growth rate decreasing like $Z^{-1 / 3} \propto k^{-1 / 3}$.

This work has been achieved under projects FTN 2003-00721 of the Spanish Ministerio de Educación y Ciencia and PAI-05-045 of the Consejería de Educación y Ciencia de la Junta de Comunidades de Castilla-La Mancha. We also thank Marie-Christine Firpo and Claude Deutsch for enriching discussions.

\section{REFERENCES}

[1] M. Tabak, J. Hammer, M. E. Glinsky, W. L. Kruer, S. C. Wilks, J. Woodworth, E. M. Campbell, M. D. Perry, and R. J. Mason. Phys. Plasmas, 1:1626, 1994.

[2] M.E. Dieckmann. Phys. Rev. Lett., 94:155001, 2005.

[3] F.A. Aharonian, A.A. Belyanin, E.V. Derishev, V.V. Kocharovsky, and Vl.V. Kocharovsky. Phys. Rev. D, 66:023005, 2002.

[4] M. Milosavljević, E. Nakar, and A. Spitkovsky. Astrophysical Journal,, 637:765, 2006.

[5] Ya. B. Faĭnberg, V.D. Shapiro, and V.I. Shevchenko. Soviet Phys. JETP, 30:528, 1970.

[6] A. Bret, M.-C. Firpo, and C. Deutsch. Nuclear Instruments and Methods in Physics Research A, 544:427, 2005.

[7] A. Bret, M.-C. Firpo, and C. Deutsch. Phys. Rev. E, 72:016403, 2005.

[8] D. A. Hammer and N. Rostoker. Phys. Fluids, 13:1831, 1970.

[9] A. Bret, M.-C. Firpo, and C. Deutsch. Phys. Rev. E, 70:046401, 2004. 